\title{
Gallstones, cholecystectomy, and the risk for developing pancreatic cancer
}

\section{ES Schernhammer*,1,2, DS Michaud ${ }^{3}$, MF Leitzmann ${ }^{4,5}$, E Giovannucci ${ }^{1,4}$, GA Colditz ${ }^{1,5,6,7}$ and CS Fuchs ${ }^{1,8}$}

'Channing Laboratory, Department of Medicine, Brigham and Women's Hospital and Harvard Medical School, Boston, Massachusetts, USA; '2Ludwig Boltzmann-Institute for Applied Cancer Research, KFJ-Spital, Vienna, Austria; ${ }^{3}$ National Cancer Institute, Nutritional Epidemiology Branch, 6120 Executive Blvd., Rockville, MD 20852, Manyland, USA; ${ }^{4}$ Department of Nutrition, Harvard School of Public Health, 665 Huntington Ave., Boston, MA 02115 , Massachusetts, USA; ${ }^{5}$ Department of Epidemiology, Harvard School of Public Health, Boston, Massachusetts, USA; ${ }^{6}$ Harvard Center for Cancer Prevention, Boston, Massachusetts, USA; ${ }^{7}$ Epidemiology Program, Dana Faber/Haward Cancer Center, Boston, Massachusetts, USA; ${ }^{8}$ Department of Adult Oncology, Dana-Farber Cancer Institute, 44 Binney Street, Boston, MA 021 I 5, Massachusetts, USA

We examined the relation between gallstones, cholecystectomy, and the development of pancreatic cancer in the Nurses' Health Study and the Health Professionals Follow-up Study. Among 104856 women and 48928 men without cancer at baseline, we documented 349 cases of pancreatic cancer during up to 16 years of follow-up. Participants were classified according to a history of gallstones or cholecystectomy. The age-adjusted relative risk of pancreatic cancer following cholecystectomy or diagnosis of gallstones was I.31 (95\% Cl, $0.93-1.83)$. However, adjustment for other pancreatic cancer risk factors attenuated the association $(R R=1.11,95 \% \mathrm{Cl}, 0.78-1.56)$; this risk did not increase with increasing time following cholecystectomy or gallstones. Gallstones or cholecystectomy do not appear to be significant risk factors for pancreatic cancer. British Journal of Cancer (2002) 86, I08I-1084. DOl: 10.1038/sj/bjc/6600193 www.bjcancer.com

(C) 2002 Cancer Research UK

Keywords: pancreatic cancer; gallstones; cholecystectomy; risk factors; prospective cohort study

Pancreatic cancer is the fifth leading cause of cancer-related mortality in the US (Greenlee et al, 2001). New environmental risk factors have consistently been linked to the risk of pancreatic cancer. Animal models suggest an important role for cytokines in experimental pancreatic carcinogenesis (Watanapa and Williamson, 1993). Cholecystokinin (CCK) has been shown to stimulate both gallbladder contraction and pancreatic enzyme secretion (Marx et al, 1987; Smith et al, 1990) and to have a trophic effect on pancreatic acinar cells (Rivard et al, 1991). The presumed increased release of CCK following cholecystectomy (Hyvarinen and Partanen, 1987) might contribute to an increased risk for pancreatic cancer risk following gallbladder removal. In addition, cholecystectomy may suppress the normal inhibitory effect of CCK on the Sphincter of Oddi (Luman et $a l, 1997)$. The presence of gallstones, on the other hand, appears to be associated with chronic pancreatitis (Hardt et al, 2001), but whether chronic pancreatitis predisposes to pancreas cancer remains controversial (Lowenfels et al, 1993; Ekbom et al, 1994). Some of the earlier studies reported relative risks of pancreatic cancer of 1.2-2.0 among individuals who had undergone a cholecystectomy previously (Haines et al, 1982; Shibata et al, 1994; Ekbom et al, 1996; Chow et al, 1999; Gullo, 1999; Silverman et al, 1999; Coughlin et al, 2000); however, the strength of the association remains uncertain because of the retrospective design of most analyses and the reliance on next-

*Correspondence: ES Schernhammer; Channing Laboratory, I8I Longwood Avenue, Boston, MA 021 I5, USA;

E-mail: eva.schernhammer@channing.harvard.edu

Received 15 October 200 I; revised I5 January 2002; accepted 19 January 2002 of-kin respondents. Prospective studies can overcome these limitations. We examined the relation between cholecystectomy or gallstones and pancreatic cancer risk in two large cohorts, the Nurses' Health Study (NHS) and the Health Professionals Follow-up Study (HPFS).

\section{MATERIALS AND METHODS}

\section{Study cohorts}

The Nurses' Health Study (NHS) enrolled 121700 female, registered nurses aged $30-55$ years in 1976, and information on their health status, medical history, and known and suspected risk factors for cancer and coronary heart disease was gathered through mailed questionnaires. The Health Professionals Follow-up Study (HPFS) began in 1986, when 51529 American male dentists, optometrists, osteopaths, pharmacists, podiatrists, and veterinarians aged 40-75 years completed a mailed questionnaire on health-related questions similar to those in NHS. Follow-up questionnaires were sent biennially to participants in both cohorts.

\section{Ascertainment of cholecystectomy or gallstone diagnosis}

In 1982, the study participants of the NHS were asked about their history of cholecystectomy and the year of surgery, with information updated biennially. History of gallstones that were not removed was also assessed on the 1982 questionnaire but was updated only through 1986. In the HPFS, participants were asked about their history of cholecystectomy or gallstones at baseline, and both questions were updated with all subsequent questionnaires. 


\section{Documentation of pancreatic cancer and deaths}

We included confirmed pancreatic cancers diagnosed between the return of the 1982 questionnaire and June 1, 1998, for the NHS and between the return of the 1986 questionnaire and January 1, 1998 , for the HPFS. With permission from study participants, we confirmed pancreatic cancer through physicians' review of their medical records. If permission was denied, we attempted to confirm the self-reported cancer with an additional letter or phone call. We also searched the National Death Index to identify deaths among the nonrespondents to each 2-year questionnaire. The computerised National Death Index is a highly sensitive method for identifying death in this cohort (Stampfer et al, 1984). For all deaths attributable to pancreatic cancer, we requested permission from family members (subject to state regulation) to review the medical records. Pancreatic cancer was considered the cause of death if the medical records or autopsy report confirmed fatal pancreatic cancer or when pancreatic cancer was listed as the underlying cause of death without another, more plausible cause. In this analysis, we included 170 cases of pancreatic cancer in the women and 127 in the men.

\section{Statistical analysis}

We excluded participants who did not answer the baseline questionnaire on gallstones and cholecystectomy (1982 in the NHS; 1986 in the HPFS) and all participants who reported a history of cancer (with the exception of non-melanoma skin cancer). Since the diagnosis of gallstones or the need for cholecystectomy might have been triggered by early symptoms associated with pancreatic cancer, we also excluded the first 2 years of follow-up for all study participants after their first report of either cholelithiasis or cholecystectomy. Therefore, for NHS participants who did not report gallstones or cholecystectomy between 1984 and 1986, we initiated follow-up with the date of return of the baseline 1982 questionnaire, whereas, for women who reported gallstones or cholecystectomy between 1984 and 1986, follow up began in 1988. In the HPFS, however, the baseline 1986 questionnaire assessed history of gallstones and cholecystectomy in timeframes that were larger than a 2-year interval. There, for all HPFS participants, we initiated follow-up with the date of return of the 1988 to ensure that persons reporting a history of either gallstones or a cholecystectomy were excluded from the succeeding 2 years of analysis only. In both cohorts, we computed person years of follow-up to the date of diagnosis of pancreatic cancer, death from any cause, or to the end of the study period (June 1, 1998, for women, and February 1, 1998 for men) whichever occurred first. After these exclusions, 104856 women and 48928 men were eligible for follow-up, and 1405681 person years were accrued in the NHS and 441387 in the HPFS.

The primary analysis used incidence rates with person-years of follow-up in the denominator. We used relative risk (RR) as the measure of association; RR was defined as the incidence rate of pancreatic cancer among participants with a history of gallstones or a cholecystectomy divided by the incidence rate among participants without such a history. In the NHS, person time with gallstones history from 1986 on was based on the cumulative history of gallstones up to 1986. For each cohort, we used Cox proportional hazards models to adjust for other potential risk factors for pancreatic cancer. Although rapid weight loss in obese patients constitutes a risk factor for gallstones (Yang et al, 1992), pancreatic cancer is also frequently associated with profound weight loss. Therefore, we did not adjust for weight change or most recent body mass index but used baseline body mass index (1976 for the NHS, 1986 for the HPFS), which appeared to be the most important weight risk factor for pancreatic cancer in these cohorts (Michaud et al, 2001). We used a random-effects model for the log of the RR (DerSimonian and Laird, 1986) to pool the data from the two cohorts.

\section{RESULTS}

A total of 206 women and 143 men were diagnosed with pancreatic cancer during 2162077 person-years of follow-up. Of these 349 persons, $333(96 \%)$ died of the disease. At baseline, 9.2\% of the women $(16.4 \%$ by 1996$)$ and $3.7 \%$ of the men $(7.1 \%$ by 1996$)$ reported gallstones or a history of cholecystectomy. Characteristics of the study population are described in Table 1.

In our primary analysis we excluded participants who reported a history of cholecystectomy or gallstones from the proceeding 2 years of analysis, which left 170 women and 127 men with a diagnosis of pancreatic cancer. Of these, women with a history of gallstones or a cholecystectomy experienced a modest elevation in risk for pancreatic cancer (age-adjusted $\mathrm{RR}=1.47$, 95\% CI, 1.00 2.16), whereas men with such a history did not experience an obvious increase in risk (Table 2). Among men and women

Table I Age and age-standardized ${ }^{\mathrm{a}}$ characteristics by exposure status (gallstones and/or cholecystectomy) among women in the NHS and men in the HPFS

\begin{tabular}{|c|c|c|c|c|}
\hline \multirow[b]{3}{*}{ Characteristics $^{\mathrm{b}}$} & \multicolumn{4}{|c|}{ Gallstones and/or cholecystectomy } \\
\hline & \multicolumn{2}{|c|}{ NHS } & \multicolumn{2}{|c|}{ HPFS } \\
\hline & Yes & No & Yes & No \\
\hline Number of individuals & 9696 & 95160 & 1799 & 47129 \\
\hline Age in years, mean (s.d.) & $50.9(6.9)$ & $48.8(7.2)$ & $60.8(9.1)$ & $54.2(9.7)$ \\
\hline Height in inches, mean (s.d.) & $64.4(3.4)$ & $64.4(3.3)$ & $69.8(3.0)$ & $70.1(2.9)$ \\
\hline Baseline body mass index $\geqslant 30(\%)$ & 20.2 & 6.8 & 16.5 & 7.8 \\
\hline History of diabetes (\%) & 6.5 & 2.6 & 5.4 & 3.1 \\
\hline Current smokers (\%) & 30.7 & 27.7 & 11.4 & 9.6 \\
\hline Average pack-years ever smoked (current or past), mean (s.d.) & $5.4(8.7)$ & $4.7(7.9)$ & | $3.0(73.9)$ & $5.0(35.1)$ \\
\hline Physical activity level in MET - hours/week ${ }^{c}$ (s.d.) & $12.2(17.5)$ & $14.3(21.4)$ & $15.9(21.2)$ & $20.3(26.3)$ \\
\hline Current $\mathrm{PMH}^{\mathrm{d}}$ use $\geqslant 25$ years $(\%)$ & 6.8 & 4.8 & N.A. & N.A. \\
\hline Nulliparous (\%) & 5.3 & 6.8 & N.A. & N.A. \\
\hline Parity $\geqslant 5$ children (\%) & 16.5 & 14.1 & N.A. & N.A. \\
\hline
\end{tabular}

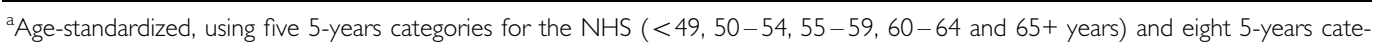
gories for the HPFS $(<49,50-54,55-59,60-64,65-69,70-74,75-79,80+$ years $)$ of age as of the 2-year period when participants first entered follow-up. 'Baseline: 1982 for NHS, 1986 for HPFS. 'MET-h/week, sum of the average time/week spent in each activity by its typical energy-expenditure requirements expressed in metabolic equivalents (METs), MET, caloric need per kilogram of body weight per hour activity, divided by the caloric need per kg per $h$ at rest. dPMH, postmenopausal hormones. 
Table 2 Adjusted relative risks (RRs) of pancreatic cancer by exposure to gallstones, cholecystectomy (CHE), and both combined (either/or) among 104856 women in the NHS and 49063 men in the HPFS, with prospective follow-up 1982 - 1998 (NHS) and 1988 - 1998 (HPFS) (total cases of pancreatic cancer, 297). Exclusion of the first 2 years of follow-up if the exposure was within 2 years of its being reported

\begin{tabular}{|c|c|c|c|c|}
\hline & $\begin{array}{c}\text { Neither cholecystectomy } \\
\text { nor gallstones }\end{array}$ & Gallstones & Cholecystectomy & $\begin{array}{l}\text { Cholecystectomy } \\
\text { or gallstones }\end{array}$ \\
\hline \multicolumn{5}{|l|}{ NHS women } \\
\hline No. of cases & 138 & 14 & 29 & 32 \\
\hline Person-years & | 255822 & 72493 & 129750 & 149859 \\
\hline Age-adjusted RR $(95 \% \mathrm{Cl})$ & 1.0 & $1.31(0.75-2.27)$ & $1.54(1.03-2.29)$ & $1.47(1.00-2.16)$ \\
\hline Multivariate RR $(95 \% \mathrm{Cl})^{\mathrm{a}}$ & 1.0 & $1.21(0.70-2.11)$ & $1.42(0.95-2.14)$ & $1.34(0.9 \mid-1.99)$ \\
\hline Multivariate RR $(95 \% \mathrm{Cl})^{\mathrm{b}}$ & 1.0 & $1.09(0.62-1.91)$ & $1.27(0.84-1.92)$ & $1.19(0.80-1.77)$ \\
\hline \multicolumn{5}{|l|}{ HPFS men } \\
\hline No. of cases & 118 & 7 & 8 & 9 \\
\hline Person-years & 419533 & 16230 & 16177 & 21854 \\
\hline Age-adjusted RR $(95 \% \mathrm{Cl})$ & 1.0 & $0.99(0.46-2.14)$ & $1.19(0.58-2.43)$ & $0.97(0.49-\mid .91)$ \\
\hline Multivariate RR $(95 \% \mathrm{Cl})^{\mathrm{a}}$ & 1.0 & $0.92(0.42-2.00)$ & $1.18(0.57-2.45)$ & $0.95(0.481 .89)$ \\
\hline Multivariate RR $(95 \% \mathrm{Cl})^{\mathrm{b}}$ & 1.0 & $0.87(0.40-1.90)$ & $1.12(0.54-2.33)$ & $0.89(0.45-1.78)$ \\
\hline \multicolumn{5}{|l|}{ Combined cohorts } \\
\hline No. of cases & 256 & 21 & 37 & 41 \\
\hline Person-years & | 675355 & 88723 & 145927 & 171713 \\
\hline Age-adjusted RR $(95 \% \mathrm{Cl})$ & 1.0 & I. $17(0.75$ I.83) & $1.45(1.02-2.07)$ & $1.31(0.93-1.83)$ \\
\hline Multivariate RR $(95 \% \mathrm{Cl})^{\mathrm{a}}$ & 1.0 & $1.10(0.70-1.73)$ & $1.36(0.95-1.94)$ & $1.23(0.88-1.74)$ \\
\hline Multivariate RR $(95 \% \mathrm{Cl})^{\mathrm{b}}$ & 1.0 & $1.00(0.64-1.59)$ & $1.23(0.86-1.77)$ & $1.11(0.78-1.56)$ \\
\hline \multicolumn{5}{|c|}{$\begin{array}{l}\text { aMultivariate risks from proportional hazards models are adjusted for age in months, follow-up cycle, history of diabetes } \\
\text { (yes/no), and smoking status in six categories (never, quit } \geqslant 15 \text { years ago, quit }<15 \text { years ago, and smoked } \leqslant 25 \text { pack }- \\
\text { years in past } 15 \text { years, quit }<15 \text { years ago and smoked }>25 \text { pack-years in past } 15 \text { years, current smoker with } \leqslant 25 \\
\text { pack-years in past } 15 \text { years, current smoker with }>25 \text { pack-years in past } 15 \text { years). } \text { bultivariate risks from propor- } \\
\text { tional hazards models are adjusted for age in months, follow-up cycle, history of diabetes, smoking status, nonvigorous } \\
\text { physical activity METs/week, in quintiles, cohort baseline (NHS: 1976, HPFS: 1986), and baseline bodymass index (I976 } \\
\text { for the NHS, } 1986 \text { for the HPFS) in five categories ( }<21,21-22.9,23-24.9,25-28.9,29-40+\text { for the NHS and } \\
<22.9,23-24.9,25-26.9,27-29.9,30+\text { for the HPFS). }\end{array}$} \\
\hline
\end{tabular}

combined, the age-adjusted RR was 1.31 (95\% CI, 0.93-1.83). However, after adjusting for other factors, including body mass index and physical activity, the RR was 1.11 (95\% CI, $0.78-$ 1.56). Adjustment for postmenopausal hormone use and parity among women did not substantially alter our estimates (data not shown).

Multivariate RRs in the analyses that included all 349 diagnosed pancreatic cancer case subjects were not substantially different from the primary analyses for women $(\mathrm{RR}=1.16,95 \% \mathrm{CI}, 0.81-1.66)$, men $(\mathrm{RR}=0.96,95 \% \mathrm{CI}, 0.52-1.75)$, or women and men combined ( $\mathrm{RR}=1.10,95 \% \mathrm{CI}, 0.81-1.50)$.

We examined the relation between time since cholecystectomy and risk of pancreatic cancer. Among individuals with a cholecystectomy 10 or fewer years in the past, the multivariate RR for pancreatic cancer was 1.12 (95\% CI, $0.66-1.89)$, whereas for more than 10 years in the past, the RR was 1.13 (95\% CI, 0.76-1.68). For cholecystectomy more than 20 years in the past, the RR was 0.88 (95\% CI, 0.49-1.59). These findings were not materially different when we examined time interval since cholecystectomy or a diagnosis of gallstones; for individuals with a history of either cholecystectomy or gallstones more than 10 years previously, the RR for pancreatic cancer was 1.16 (95\% CI, 0.79-1.70).

\section{DISCUSSION}

In two prospective cohorts of women and men, risk for pancreatic cancer following diagnosis of gallstones or cholecystectomy was modestly but not significantly increased. This risk was attenuated substantially after adjustment for potential confounders, including body mass index and physical activity. Furthermore, the risk did not increase in the decades following either cholecystectomy or diagnosis of gallstones.

The prospective nature of our studies precluded recall bias and the need of next-of-kin respondents. Moreover, to avoid misclassification of exposure, we updated reports of cholecystectomy and gallstones biennially. Because identification of deaths is highly accurate in these cohorts (Stampfer et al, 1984), differential follow-up is unlikely. Nonetheless, case number may have compromised the ability to explore further details in these cohorts. Based on the available statistical power, we cannot completely exclude an effect for cholecystectomy or cholelithiasis, although in both cohorts the effect appears to be consistently small. It is possible that gallstones and pancreatic cancer have similar etiologic factors (e.g. hyperinsulinemia), which may account for an association between the two. Controlling for physical activity and BMI, a strong determinant of hyperinsulinaemia, reduced the modest association between cholecystectomy and pancreatic cancer.

Previous studies have investigated a potential association between gallstones or cholecystectomy and risk of pancreatic cancer. Results from case-control studies have been conflicting (Haines et al, 1982; Shibata et al, 1994; Ekbom et al, 1996; Coughlin et al, 2000), although some indicate a $30-70 \%$ increased risk of pancreatic cancer in association with gallstones or after a cholecystectomy (Chow et al, 1999; Gullo, 1999; Silverman et al, 1999; Silverman, 2001). In the largest case-control study, Silverman (2001) observed a RR of $1.7(95 \% \mathrm{CI}, 1.0-1.3)$. One prospective study found an increased RR for pancreatic cancer of $1.99(95 \%$ CI, 1.08-3.68) associated with cholecystectomy after adjustment for sex, age, and cigarette smoking (Shibata et al, 1994). However, that study did not adjust for potential confounders such as obesity and was limited by a small number of cases. The only other cohort study observed a slightly elevated risk for pancreatic cancer in men with a history of gallstones (RR 1.2, 95\% CI, 1.0-1.5), but their ability to control for potential confounding factors in this large cohort was compromised by incomplete follow-up (Coughlin et $a l, 2000)$. Few studies have examined the time interval between cholecystectomy and incidence of pancreatic cancer. Silverman (2001) found a $70 \%$ excess risk of pancreatic cancer 20 or more years after a cholecystectomy. In contrast, Hyvarinen and Partanen found a statistically significant increased risk of pancreatic cancer 
within 5 years after cholecystectomy $(P=0.007)$ but not thereafter (Hyvarinen and Partanen, 1987). Moreover, Ekbom et al (1996) found no increase in risk with increased duration after cholecystectomy.

Although the age-adjusted findings in our two cohorts are somewhat more consistent with previous positive results, multivariate adjustment suggests considerable confounding in this relation.

In summary, our findings do not support a strong relation between gallstones or prior cholecystectomy and pancreatic cancer risk. The effect of gallstones and cholecystectomy on pancreatic cancer risk appears small and residual confounding by risk factors for both cholecystectomy and pancreatic cancer may have explained the results in previous studies.

\section{REFERENCES}

Chow WH, Johansen C, Gridley G, Mellemkjaer L, Olsen J, Fraumeni JFJ (1999) Gallstones, cholecystectomy and risk of cancers of the liver, biliary tract and pancreas. Br J Cancer 79: 640-644

Coughlin SS, Calle EE, Patel AV, Thun MJ (2000) Predictors of pancreatic mortality among a large cohort of United States adults. Cancer Causes Control 11: $915-923$

DerSimonian R, Laird N (1986) Meta-analysis in Clinical Trials. Controlled Clin Trials 7: $177-188$

Ekbom A, McLaughlin JK, Karlsson BM, Nyren O, Gridley G, Adami HO, Fraumeni JFJ (1994) Pancreatitis and pancreatic cancer: a populationbased study. J Natl Cancer Inst 86: 625-627

Ekbom A, Yuen J, Karlsson BM, McLaughlin JK, Adami HO (1996) Risk of pancreatic and periampullar cancer following cholecystectomy: a population-based cohort study. Dig Dis Sci 41: 387-391

Greenlee RT, Hill-Harmon MB, Murray T, Thun M (2001) Cancer Statistics,. (2001.) CA Cancer J Clin 51: 15-36

Gullo L (1999) Risk of pancreatic and periampullary cancer following cholecystectomy. Ann Oncol 10: $127-128$

Haines AP, Moss AR, Whittemore A, Quivey J (1982) A case-control study of pancreatic carcinoma. Cancer Res Clin Oncol 103: 93-97

Hardt PD, Bretz L, Krauss A, Schnell-Kretschmer H, Wusten O, Nalop J, Zekorn T, Klor HU (2001) Pathological pancreatic exocrine function and duct morphology in patients with cholelithiasis. Dig Dis Sci 46: $536-539$

Hyvarinen H, Partanen S (1987) Association of cholecystectomy with abdominal cancers. Hepatogastroenterology 34: 280-284

Lowenfels AB, Maisonneuve P, Cavallini G, Ammann RW, Lankisch PG, Andersen JR, Dimagno EP, Andren-Sandberg A, Domellof L (1993) Pancreatitis and the risk of pancreatic cancer. International Pancreatits Study Group. N Engl J Med 328: $1433-1437$

Luman W, Williams AJ, Pryde A, Smith GD, Nixon SJ, Heading RC, Palmer KR (1997) Influence of cholecystectomy on sphincter of Oddi motility. Gut 41: $371-374$

\section{ACKNOWLEDGEMENTS}

This research was supported by National Cancer Institute Grants CA/ES62984 and CA87969, as well as CA 40356, CA 55075, and CA 86102. Dr Schernhammer was supported in part by a Research Grant in Cancer Prevention from the Austrian Federal Ministry of Education, Science and Culture. We are indebted to the participants of the Nurses' Health Study for their continuing outstanding dedication to the study and to Karen Corsano for technical assistance.
Marx M, Gomez G, Lonovics J, Thompson JC (1987) Cholecystokinin. In Gastrointestinal Endocrinology Thompson JC, Greeley Jr GH, Rayford PL, Townsend Jr CM (eds) pp 213-222 New York: McGraw-Hill

Michaud DS, Giovannucci E, Willett WC, Colditz GA, Stampfer MJ, Fuchs CS (2001) Physical activity, obesity, height, and the risk of pancreatic cancer. JAMA 286: $921-929$

Rivard N, Guan D, Maouyo D, Grondin G, Berube FL, Morisset J (1991) Endogenous cholecystokinin release responsible for pancreatic growth observed following pancreatic juice diversion. Endocrinology 129: $2867-$ 2874

Shibata A, Mack TM, Paganini-Hill A, Ross RK, Henderson BE (1994) A prospective study of pancreatic cancer in the elderly. Int $J$ Cancer 58: $46-49$

Silverman DT (2001) Risk factors for pancreatic cancer: a case-control study based on direct interviews. Teratog Carcinog Mutagen 21: 7-25

Silverman DT, Schiffman M, Everhart J, Goldstein A, Lillemoe KD, Swanson GM, Schwartz AG, Brown LM, Greenberg RS, Schoenberg JB, Pottern LM, Hoover RN, Fraumeni JFJ (1999) Diabetes mellitus, other medical conditions and familial history of cancer as risk factors for pancreatic cancer. $\mathrm{Br}$ J Cancer 80: 1830 - 1837

Smith JP, Salomon TE, Bagheri S, Kramer S (1990) Cholecystokinin stimulates growth of human pancreatic adenocarcinoma. Dig Dis Sci 35: $1377-1384$

Stampfer MJ, Willett WC, Speizer FE, Dysert DC, Lipnick R, Rosner B, Hennekens $\mathrm{CH}$ (1984) Test of the national death index. Am J Epidemiol 119: $837-839$

Watanapa P, Williamson RCN (1993) Experimental pancreatic hyperplasia and neoplasia: effects of dietary and surgical manipulation. $\mathrm{Br} J$ Cancer 67: $877-884$

Yang H, Petersen GM, Roth MP, Schoenfield LJ, Marks JW (1992) Risk factors for gallstone formation during rapid weight loss. Dig Dis Sci 37: $912-918$ 